\title{
Methotrexate efficacy and tolerability after switching from oral to subcutaneous route of administration in juvenile idiopathic arthritis
}

\author{
Zbigniew Żuber, Dorota Turowska-Heydel, Małgorzata Sobczyk, Marta Banach-Górnicka, \\ Katarzyna Rusnak, Anna Piszczek, Elżbieta Mężyk
}

Department of Older Children with subunits of Neurology, Rheumatology and Rehabilitation, St. Louis Regional Specialised Children's Hospital, Krakow, Poland

\begin{abstract}
Objectives: Methotrexate (MTX) is one of the most frequently used, highly effective disease-modifying drugs in juvenile idiopathic arthritis (JIA) therapy. The drug can be administered orally or subcutaneously, but the efficacy and tolerance of these two routes of administration raise doubts in JIA patients. The aim of the study was to evaluate MTX efficacy and tolerability after switching from the oral to the subcutaneous route of administration in children with JIA.

Material and methods: A single-centre, questionnaire-based assessment of MTX efficacy and tolerance in 126 unselected JIA patients with longer than 6 months of follow-up was performed. In all patients, MTX was initially administered orally. The response to MTX treatment was analysed according to American College of Rheumatology (ACR) paediatric criteria.

Results: Six-month MTX therapy was effective (ACR score $\geq 30$ ) in 83 children (65.9\%). The oral route of MTX administration was changed to subcutaneous in 32 patients after a mean period of 14 months due to intolerance $(n=20)$ or reluctance to take the oral formulation $(n=12)$. This group of children was significantly younger $(p=0.02)$ but did not differ from the group of children that continued oral treatment in other aspects, including MTX dose.

Six months after switching from oral to subcutaneous MTX the ACR score remained unchanged. Three children (9.4\%) still reported symptoms of drug intolerance.

Conclusions: The switch from oral to subcutaneous MTX may increase the response rate in JIA patients with intolerance of its oral formulation. The reluctance to take oral MTX can be anticipated in early childhood, and should be considered in the individualization of therapy, having also in mind the lower risk of severe gastrointestinal adverse drug reactions.
\end{abstract}

Key words: juvenile idiopathic arthritis, methotrexate, subcutaneous administration, oral administration.

\section{Introduction}

Juvenile idiopathic arthritis (JIA) is one of the most common groups of autoimmune diseases in the developmental period. The diagnosis of JIA is established when symptoms of arthritis persist for more than 6 weeks, primarily after the exclusion of all secondary forms of arthritis, in children aged up to 16 years [1-3]. The current classification system by the International League Against Rheumatism - ILAR (Edmonton 2001) distinguishes seven JIA categories, characterized by different clinical presentation and laboratory findings, as well as the outcome: systemic arthritis, oligoarthritis

Address for correspondence:

Zbigniew Żuber, Department of Older Children with subunits of Neurology, Rheumatology and Rehabilitation, St. Louis Regional Specialised Children's Hospital, Strzelecka 2, 31-503 Kraków, tel. +48 1261986 30, fax +48 1261986 81, e-mail: zbyszekzuber@interia.pl Submitted: 27.01.2016; Accepted: 26.02.2016 
(persistent, extended), polyarthritis, rheumatoid factor positive $[R F(+)]$, polyarthritis, rheumatoid factor negative $[R F(-)]$, enthesitis-related arthritis (JIA-ERA), psoriatic arthritis (JIA-PA), and unclassified arthritis [2].

The aetiology of JIA remains unknown. Gender and loci of human leukocyte antigen (HLA) classes I and II were found to be significant factors influencing the development and clinical course of the disease. The pathomechanism of chronic inflammation in JIA involves, inter alia, excessive activation of NK cells, T lymphocytes and macrophages, and uncontrolled release of proinflammatory cytokines affecting regulation of the immune response [3].

Therapy of IIA is determined by the category of the disease, its onset, activity and the occurrence of poor prognosis factors [4]. Non-steroidal anti-inflammatory drugs (NSAIDs), disease-modifying drugs (DMARDs), glucocorticoids (GCS), immunosuppressive agents, and biological agents are currently recommended. Long-term treatment with DMARDs should be started shortly after the diagnosis of JIA is made. The first-line drug and the gold standard for the treatment of JIA is methotrexate (MTX) - an inhibitor of dihydrofolate reductase (DHFR), which is an enzyme that participates in synthesis of tetrahydrofolate, a cofactor in the synthesis of purines. It is administered in a weekly dose of $10-30 \mathrm{mg} / \mathrm{m}^{2}$ of body surface area (BSA) either in oral or subcutaneous formulation in children [5]. The drug has been used in JIA treatment since the 1980s, and more recently gained importance in the treatment of other rheumatic diseases in children [6]. An anti-inflammatory effect of MTX, the therapeutic target in rheumatology, is achieved by using doses much lower than those necessary for the effective anti-proliferative treatment in oncology. The main mechanism of anti-inflammatory action is attributed to the modulation of the adenosine pathway followed by the increase in intracellular CAMP level resulting in inhibition of phagocytosis and secretion of interferon, IL-6, IL-12 and tumour necrosis factor (TNF) [5, 7].

The results of performed studies indicate a response to MTX treatment in approximately $70 \%$ of JIA patients. A response can be more frequently achieved in carriers of rs1045642 polymorphic variant for the adenosine triphosphate-binding cassette transporter B1 (ABCB1) gene and rs4793665 for the adenosine triphosphate-binding cassette transporter $\mathrm{C} 1$ (ABCC3) gene, but less frequently in those with the rs1051266 variant for the solute carrier 19A1 (SLC19A1) gene [8] and with greater concentrations of myeloid-related protein 8 and 14 (MPR8/14), surrogate markers of residual synovial inflammation [8, 9]. In addition, due to the limited bioavailability of MTX during enteral use, subcutaneous administration is recommended in JIA therapy if higher doses are required.
Rarely MTX doses in JIA patients have to be tailored to kidney excretory function as the drug is mostly excreted unchanged by the kidneys (approximately 80\%), and to a lesser extent with bile (approximately 20\%) [10].

The most commonly observed adverse drug reactions (ADRs) in children include gastro-intestinal symptoms (abdominal pain, nausea, vomiting), elevated liver enzymes, mouth ulcers, restlessness, and crying [11]. It is estimated that ADRs may occur even in $75-95 \%$ of those treated, although their severity is mostly mild and rarely necessitates a switch of the route of administration or treatment discontinuation [12-14]. Methotrexate is still considered as the safest DMARD currently applied in rheumatic diseases [5].

The aims was to evaluate MTX efficacy and tolerability after switching from the oral to the subcutaneous route of administration in children with JIA.

\section{Material and methods}

We conducted a questionnaire-based assessment of efficacy and tolerance of MTX administered orally or subcutaneously in the routine treatment in unselected patients diagnosed with JIA from January 2010 to December 2013, treated in the Department of Older Children with subunits of Neurology, Rheumatology and Rehabilitation, St. Louis Regional Specialised Children's Hospital in Cracow (Poland). The study was performed from October 2010 to November 2014.

\section{Assessment of treatment efficacy and drug tolerance}

The response to MTX treatment was analysed according to American College of Rheumatology (ACR) paediatric criteria. In order to eliminate the effect of small fluctuations in the sedimentation rate (ESR) on the ACR result we introduced the following classification of ESR: < $20 \mathrm{~mm} / \mathrm{h}-1$ point; 20-39- 2 points; 40-693 points; 70-99 -4 points; $>100-5$ points.

Methotrexate intolerance (the occurrence of ADRs) was scored according to a questionnaire (Table I) completed by children and their parents. The tolerance was scored as very good (0 points), good (1 point), moderate (2-3 points), or bad (4-5 points).

\section{Statistical analysis}

The analyses were performed using the STATISTICA 10.0 PL for Windows software package (StatSoft Polska, Kraków, Poland). Values are presented as median values with 25-75 percentiles. For comparison of groups, we used the $\chi^{2}$ test and Fisher test (qualitative variables) and the Mann-Whitney $U$ test (quantitative variables). In 
Table I. Questionnaire for oral methotrexate (MTX) tolerance used in the study

\begin{tabular}{|c|c|}
\hline 1. Does the child take medication orally? & Yes/No \\
\hline 2. Is there any problem with oral MTX use? & Yes/No \\
\hline \multicolumn{2}{|c|}{ If yes, what is the problem with oral MTX use? } \\
\hline Abdominal pain & Yes (1) / No (0) \\
\hline Nausea & Yes (1) / No (0) \\
\hline Vomiting & Yes (1) / No (0) \\
\hline Behavioural problems & Yes (1) / No (0) \\
\hline Other (please specify) & Yes (1) / No (0) \\
\hline $\begin{array}{l}\text { Refusal of oral administration by the } \\
\text { child }\end{array}$ & Yes (1) / No (0) \\
\hline
\end{tabular}

all statistical tests $p$-values below 0.05 were considered statistically significant.

\section{Results}

In the 4-year period, JIA was diagnosed in 150 children, of whom 2 patients did not receive MTX. Eighteen patients were lost to follow-up within 6 months from the time of diagnosis. An adverse drug reaction (subcutaneous lumps) was observed in 1 patient, and caused MTX therapy cessation. Additionally, the therapy was discontinued temporarily in 2 and permanently in $1 \mathrm{pa}$ tient due to increased activity of aminotransferases.

The characteristics of the study group of $126 \mathrm{pa}$ tients are presented in Table II. The median time from onset of symptoms to diagnosis was 3 (2-7) months (range 1.5 to 60).

The mean initial oral MTX dose was $12.6 \mathrm{mg} / \mathrm{m}^{2}$ of body surface area (BSA). Methotrexate was initially co-administered with steroids in 47 patients (37.3\%) in a mean dose of prednisolone (or its equivalent) of $0.36 \mathrm{mg} / \mathrm{kg}$ of body mass (range from 0.06 to 0.71 ). Sixmonth MTX therapy was effective (ACR score $\geq 30$ ) in 83 children (65.9\%). In 40 patients the ACR score was equal to or greater than 70 .

The tolerance of oral MTX was very good in 25 (26.6\%), good in 51 (54.3\%) and moderate in 18 (19.1\%). In 32 patients (22 girls and 10 boys) the tolerance was bad $(n=20)$ or children reported reluctance to take oral medication, either in general or MTX specifically $(n=12)$. Among 20 patients with MTX intolerance, 18 reported ADRs, mostly related to the gastrointestinal tract (abdominal pain $-n=8$, nausea $-n=6$ and vomiting $n=4)$, and $2(6.25 \%)$ complained of dizziness and weakness. In this group, the route of MTX administration was changed to subcutaneous after a mean period of 14 months (range 1 to 48). These children were signifi-
Table II. Study group characteristics

\begin{tabular}{|lc|}
\hline Gender [girls/boys] & $80 / 46$ \\
\hline Age at symptom onset [years] & $6(2-11) ;$ range 1-17 \\
\hline Age at diagnosis [years] & $7(3-12) ;$ range 1-18 \\
\hline JIA categories & {$[n, \%]$} \\
\hline Oligoarthritis & $64,50.8$ \\
\hline Polyarthritis RF(-) & $45,35.7$ \\
\hline Polyarthritis RF(+) & 0 \\
\hline Systemic arthritis & $11,8.7$ \\
\hline Enthesitis-related arthritis & $4,3.2$ \\
\hline Psoriatic arthritis & 0 \\
\hline Unclassified & $2,1.6$ \\
\hline Concomitant therapy & {$[n, \%]$} \\
\hline Sulfasalazine & $32,25.4$ \\
\hline Cyclosporine A & $18,14.3$ \\
\hline Glucocorticoids & $47,37.3$ \\
\hline Azathioprine & $2,1.6$ \\
\hline Hydroxychloroquine & $4,3.2$ \\
\hline
\end{tabular}

cantly younger [median age at diagnosis 3 (2-7) vs. 8 (4-12) years; $p=0.02$ ). The initial subcutaneous MTX dose was $12.8 \mathrm{mg} / \mathrm{m}^{2}$ of BSA, weekly. No other significant differences were found between the group of children that continued treated with oral MTX and those switched to the subcutaneous form. There was similar distribution of gender $(p=0.91)$, disease categories $(p=0.99)$, response to treatment after 6 months (ACR score) $(p=0.99)$, period of time from symptom onset to diagnosis $(p=0.22)$, and MTX dose $(p=0.73)$.

At the time of change only 2 children were receiving steroids. Six months after switching from oral to subcutaneous MTX, the clinical status (ACR score) remained unchanged ( $p=0.89)$. Twelve children $(37.5 \%)$ had improved, and 12 (37.5\%) got worse. Only 3 children (9.4\%) still reported ADRs, mostly from the gastrointestinal tract (nausea and vomiting).

\section{Discussion}

The results of our study demonstrate that more than $30 \%$ of JIA patients with intolerance or reluctance to take oral MTX benefit from switching to the subcutaneous route of administration, and suggest that the use of the subcutaneous formulation is associated with fewer gastrointestinal ADRs. It should be emphasised that the study design precluded a direct comparison of oral and 
subcutaneous MTX administration, in the aspect of both therapeutic efficacy and drug tolerance.

The structure of JIA categories in the analysed group does not reflect their prevalence in epidemiological studies [15], as children with JIA-ERA are frequently on sulfasalazine monotherapy. Therefore, the predominant group consisted of patients with oligoarthritis (54\%) and polyarthritis (36\%). This also limits the reliability of therapeutic efficacy assessment to these two subgroups.

Six-month MTX therapy was effective in $65.9 \%$ of patients, and no further improvement was observed after switching to the subcutaneous route of administration. Also other authors suggest similar efficacy of oral and subcutaneous MTX administration. Klein et al. [16] reported similar efficacy of oral and subcutaneous MTX in the German Methotrexate Registry of JIA patients. After 6 months of treatment a clinical response, according to the ACR 30 score, was obtained by $73 \%$ and $72 \%$ of patients. The discontinuation rate caused by adverse events was twice as high in patients with subcutaneous MTX application (11\% vs. 5\%; $p=0.02$ ) [16]. Also, a Netherlands study showed more frequent intolerance of parenteral than oral MTX (67.5\% vs. $44.5 \%)$ in JIA patients, related to the occurrence of behavioural symptoms, e.g. restlessness, crying and irritability, but not to gastrointestinal ADRs. Of those with oral MTX intolerance, $73.5 \%$ reported abdominal pain, 91.8\% nausea, and $45.9 \%$ vomiting [11]. In contrast, Rutkowska-Sak et al. [17] reported higher risk of vomiting and diarrhoea among patients with rheumatoid arthritis receiving oral compared to subcutaneous MTX. Additionally, the intensity of gastrointestinal side effects was related to the MTX dose [17]. Similarly, a German study found a significant correlation between the oral route of administration of MTX and the occurrence of ADRs from the gastrointestinal tract (especially nausea, vomiting, abdominal pain, and loss of appetite) for adult patients treated for rheumatoid arthritis. Frequency of ADRs among the patients taking the drug subcutaneously was significantly reduced [16]. Our data cannot be directly compared, as the subcutaneous MTX tolerance was assessed only in patients with oral MTX intolerance or reluctance to take its oral formulation. However, they show that in the majority of patients with oral MTX intolerance, mostly due to gastrointestinal ADRs, the subcutaneous formulation is well tolerated. Patients with oral and subcutaneous MTX intolerance as well as non-responders require the use of other DMARDs or biological anticytokine therapy.

The quality of available evidence concerning comparison of tolerance between oral and subcutaneous MTX in JIA patients is low. Randomised studies are lacking. It should be stressed that it is difficult to avoid selection bias in observational studies and registries.
Additionally, there is some evidence suggesting different MTX bioavailability after oral and subcutaneous administration. The results presented by Tuková et al. [18] suggest saturable intestinal absorption of oral MTX that may limits its bioavailability (and efficacy) within the range of standard doses used to treat children with JIA. Furthermore, the assignation of ADRs in combined therapy, which is frequently required, to the single drug, may be difficult and doubtful in some cases.

\section{Conclusions}

In summary, the switch from oral to subcutaneous methotrexate may increase the response rate in JIA patients with intolerance of its oral formulation. The reluctance to take oral MTX can be anticipated in early childhood, and should be considered in the individualization of therapy, having also in mind the lower risk of severe gastrointestinal adverse drug reactions.

The authors declare no conflict of interest.

\section{References}

1. Cassidy JT, Petty RE. Juvenile idiopathic arthritis. In: Textbook of pediatric rheumatology. Cassidy JT, Petty RE (eds.). $5^{\text {th }}$ ed. Saunders, Philadelphia 2005; 291-303.

2. Petty RE, Southwood TR, Manners P, et al. International League of Associations for Rheumatology classification of JIA: second revision, Edmonton 2001. J Rheumatol 2004; 31: 390-392.

3. Rutkowska-Sak L. Juvenile idiopathic arthritis - not only news. Termedia, Poznań 2014.

4. Rutkowska-Sak L, Majdan M, Tłustochowicz M, Tłustochowicz W. Diagnosis and terapeutic guidelines for chronic arthritis - the transition from paediatric rheumatologist to a rheumatologist adults. Reumatologia 2013; 51: 259-264.

5. Tłustochowicz W (ed.). Methotrexate - application in rheumatology and dermatology. 2nd ed. Termedia, Poznań 2014.

6. Kaltsonoudis E, Papagoras C, Drosos A. Current and future role of methotrexate in the Therapeutic Armamentarium for Rheumatoid Arthritis. Int J Clin Rheumatol 2012; 7: 179-189.

7. Cutolo M, Sulli A, Pizzorni C, et al. Anti-inflammatory mechanisms of methotrexate in rheumatoid arthritis. Ann Rheum Dis 2001; 60: 729-735.

8. de Rotte MC, Bulatovic M, Heijstek MW, et al. ABCB1 and ABCC3 Gene polymorphisms, are associated with first-year response to methotrexate in juvenile idiopathic arthritis. J Rheumatol 2012; 39: 2032-2040.

9. Foell D, Frosch M, Schulze zur Wiesch A, et al. Methotrexate treatment in juvenile idiopathic arthritis: when is the right time to stop? Ann Rheum Dis 2004; 63: 206-208.

10. Songsiridej N, Furst DE. Methotrexate - the rapidly acting drug. Baillieres Clin Rheumatol 1990; 4: 575-593.

11. Bulatović M, Heijstek MW, Verkaaik M, et al. High prevalence of methotrexate intolerance in juvenile idiopathic arthritis: 
development and validation of a methotrexate intolerance severity score. Arthritis Rheum 2011; 63: 2007-2013.

12. Salliot C, van der Heijde D. Long term safety od methotrexate monotherapy in patients with rheumatoid arthritis: a systematic literature research. Ann Rheum Dis 2009; 68: 1100-1104.

13. Verstappen SM, Bakker MF, Heurkens AH, et al. Adverse events and factors associated with toxicity in patients with early rheumatoid arthritis treated with methotrexate tight contro therapy: The CAMERA study. Ann Rheum Dis 2010; 69: 1044 1048.

14. Yazici Y, Sokka T, Kautiainen $\mathrm{H}$, et al. Long term safety of methotrexate in routine clinical care: discontinuation is unusual and rarely the result of laboratory abnormalities. Ann Rheum Dis 2005; 64: 207-211.

15. Żuber Z, Kania U, Król-Zdechlikiewicz A, et al. Analysis of clinical symptoms and laboratory profiles in children with juvenile idiopathic arthritis in Malopolska Region (Poland) in the Years 2007-2010. Maced J Med Sci 2014; 7: 56-61.

16. Klein A, Kaul I, Foeldvari I, et al. Efficacy and safety of oral and parenteral methotrexate therapy in children with juvenile idiopathic arthritis: an observational study with patients from the German Methotrexate Registry. Arthritis Care Res (Hoboken) 2012; 64: 1349-1356.

17. Rutkowska-Sak L, Rell-Bakalarska M, Lisowska B. Oral vs. subcutaneous low-dose methotrexate treatment in reducing gastrointestinal side effects. Reumatologia 2009; 47: 207-211.

18. Tuková J, Chládek J, Nemcová D, et al. Methotrexate bioavailability after oral and subcutaneous dministration in children with juvenile idiopathic arthritis. Clin Exp Rheumatol 2009; 27: 1047-1053. 